\title{
A gate sizing and transistor fingering strategy for subthreshold CMOS circuits
}

\author{
Morteza Nabavi $^{\mathrm{a})}$ and Maitham Shams ${ }^{\mathrm{b}}$ ) \\ Department of Electronics, Carleton University, \\ 1125 Colonel By Drive, Ottawa, Ontario K1S 5B6, Canada \\ a)nabavi@doe.carleton.ca \\ b)shams@doe.carleton.ca
}

\begin{abstract}
Parallel Transistor Stacks (PTS) has been shown to be an effective technique for improving the speed of digital circuits operating in the subthreshold region which comes at the cost of power consumption and area. However, our experience shows that using PTS is not beneficial in all cases. In this paper, we present a methodology to identify whether using PTS is beneficial (or not) in a particular CMOS technology and what transistor sizing can be employed to maximize the circuit speed. Our technique is based on analyzing the Current-OverCapacitance (COC) ratio of PMOS and NMOS transistors. The results of incorporating the proposed methodology in a 4-bit comparator and a 19-stage inverter ring oscillator, using $90 \mathrm{~nm}$ CMOS technology, illustrate $26 \%$ and $40 \%$ extra improvement compared to the blind use of PTS, respectively.
\end{abstract}

Keywords: VLSI, CMOS, logic design, subthreshold circuits

Classification: Integrated circuits

\section{References}

[1] B. H. Calhoun, A. Wang, and A. P. Chandrakasan, "Modeling and Sizing for Minimum Energy Operation in Sub-threshold Circuits," IEEE J. SolidState Circuits, vol. 40, no. 9, pp. 1778-1786, Sept. 2005.

[2] M. Muker and M. Shams, "Designing digital subthreshold CMOS circuits using parallel transistor stacks," IET Electronics Letter, vol. 47, no. 6, pp. 372-374, March 2011. Also see the featured interviews on page 354 in the same issue.

[3] J. M. Rabaey, A. Chandrakasn, and B. Nikolic, Digital Integrated Crcuits: A Design Perspective, 2nd ed., Pearson Education, Toronto, 2003.

\section{Introduction}

Digital circuits operating in the subthreshold region benefit from very low power consumption at the cost of speed [1]. In order to improve the speed of subthreshold circuits, Muker and Shams [2] introduced the Parallel Transistor Stacks (PTS) structure that, for example, enhances the speed of a 9-stage 
ring oscillator by 2.63 times compared to the conventional transistor sizing optimization method at a supply voltage of $0.3 \mathrm{~V}$ in a $65 \mathrm{~nm}$ LP CMOS technology. However, there are CMOS technologies in which using PTS blindly may not improve the speed of a subthreshold circuit. In this paper, we describe a methodology to identify the case when using PTS is beneficial for improving the speed of a digital circuit operating in the subthreshold region. We also elaborate on obtaining the appropriate transistor sizing where applying PTS is advised.

\section{Propagation delay}

The propagation delay $(D)$ for charging or discharging a node is given by [3]:

$$
D=\frac{C V_{D D}}{2 I},
$$

where $C$ is the load capacitance, $I$ denotes the average current, and $V_{D D}$ is the supply voltage. As (1) indicates, higher speeds (i.e., lower propagation delays) can be achieved by increasing the current and lowering the capacitance. Therefore, the highest operating frequency is attained when the CurrentOver-Capacitance (COC) ratio is maximized. This is often exactly or close to the transistor's minimum width in modern nanometer CMOS technologies, as a result of the so-called Inverse-Narrow-Width-Effect (INWE) [2].

Fig. 1 shows the COC ratio versus the width of both PMOS and NMOS transistors in a $130 \mathrm{~nm}$ CMOS technology. The transistors are biased for maximum current drive in the subthreshold region (i.e., $V_{D S}=\left|V_{G S}\right|$ ). The capacitance includes the total gate and junction capacitance of the transistor. Based on this figure, in order to achieve a higher COC ratio we either use the minimum transistor width (point A) or use PTS locked to the minimum width. Nevertheless, the maximum COC ratio is not always associated with the minimum transistor width, as shown in Fig. 1 (b). The COC ratio for the PMOS transistor shown in this figure consists of two regions. In Region 1

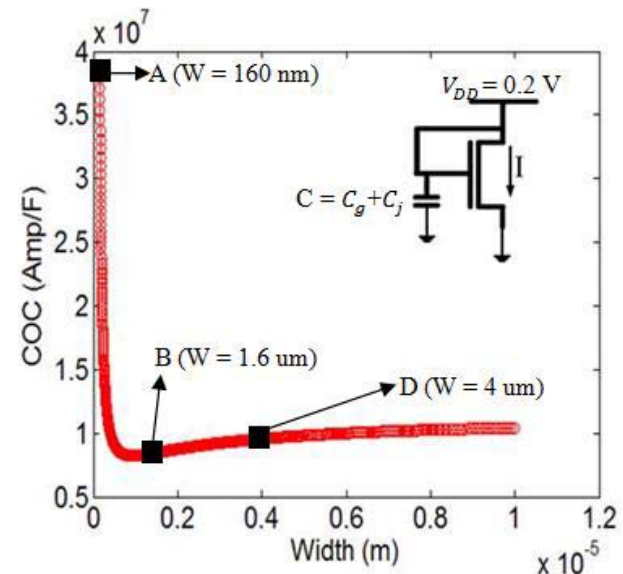

(a)

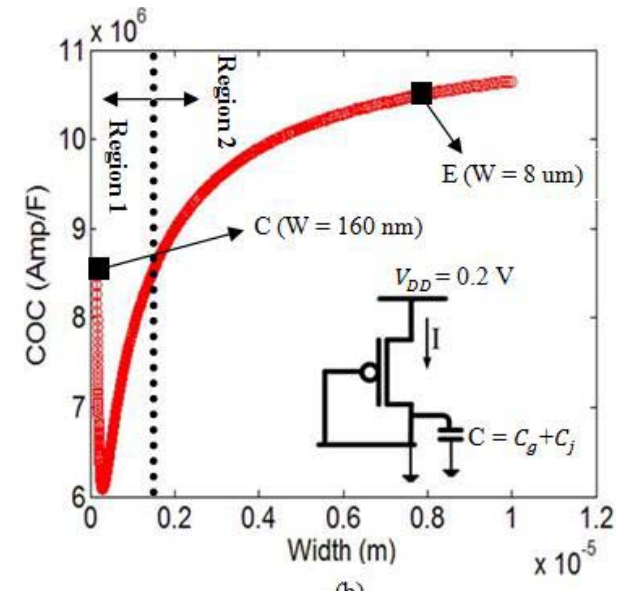

(b) (c) IEICE 2012

DOI: $10.1587 /$ elex.9.1550 Received June 16, 2012 Accepted August 09, 2012 Published October 11, 2012

Fig. 1. Current over capacitance ratio as a function of width for transistors in a $130 \mathrm{~nm}$ CMOS technology: a) NMOS, b) PMOS. 
the COC ratio corresponding to the widths larger than the minimum width have a lower value compared to the COC ratio at the minimum width. On the other hand, the widths located in Region 2 have a COC ratio larger than the minimum width. Based on this figure, we use PTS for transistor widths in Region 1 and avoid PTS for transistor widths in Region 2. Applying PTS on the transistors in Region 2 will result in a lower circuit speed.

\section{Methodology}

The following steps explain our methodology on how to identify the cases when to use and when not to use PTS in designing a logic circuit.

1- Plot the COC ratio versus width for each transistor type (PMOS and NMOS) for the given technology kit (e.g., Fig. 1).

2- Based on Step 1, identify the transistor width $\left(W_{I N W E}\right)$ associated with the local maximum COC ratio, which typically appears near the minimum width due to INWE [2]. As shown in Fig. 1, point A denotes $W_{I N W E}$ for NMOS transistor and point C denotes $W_{I N W E}$ for PMOS transistor.

3- Apply a transistor sizing optimization method such as Logical Effort [3] to optimize the circuit performance.

4- Find the optimum width $\left(W_{\text {opt }}\right)$ for each transistor in the circuit based on Step 3.

5- Compare $C O C_{\text {opt }}$, the COC ratio corresponding to $W_{\text {opt }}$ (Step 4), with $C O C_{I N W E}$, the COC ratio corresponding to $W_{I N W E}$ (Step 2).

a. If $C O C_{o p t}<C O C_{I N W E}$, use PTS, and split the transistor of width $W_{o p t}$ into multiple $(K)$ transistors of width $W_{I N W E}$, where $K=W_{\text {opt }} / W_{I N W E}$ rounded to the nearest digit. For example, if Logical Effort finds that an NMOS transistor should have a width of $1.6 \mu \mathrm{m}$ (point B in Fig. 1(a)) we split it into $K=$ $W_{\text {opt }} / W_{\text {INWE }}=10$ transistors at the width of $160 \mathrm{~nm}$ (point $\mathrm{A}$ in Fig. $1(\mathrm{a}))$.

b. Else If $C O C_{o p t} \geq C O C_{I N W E}$, using PTS results in a slower circuit. In this case we keep the transistor width at $W_{\text {opt }}$. For example, if Logical Effort finds a transistor of width $8 \mu \mathrm{m}$ (point E in Fig. 1(b)), we don't use PTS, as the COC ratio at point E has already a higher value compared to the COC ratio at $160 \mathrm{~nm}$ (point C in Fig. 1(b)).

Note that Logical Effort (LE) is a much simpler optimization method compared to exhaustive blind simulations. Although LE may not result in the exact optimum solution, especially after extracting layout parasitic, it gives a very good initial point to start with. Iterative applications of LE may be 
needed for fine tuning. However, LE assumes that the current of a transistor is linearly proportional to its width. This assumption is not valid for MOSFETs operating in the subthreshold region in most modern CMOS technologies due to the INWE [2]. By using PTS, a linear-relationship between the current of the transistor and its width is established, enabling one to use LE for transistor sizing of subthreshold circuits [2].

\section{Results}

A 19-stage inverter ring oscillator running at $0.2 \mathrm{~V}$ has been examined to verify the correctness of the proposed methodology in $130 \mathrm{~nm}$ and $90 \mathrm{~nm}$ CMOS technologies. We explored the effect of incorporating PTS on each inverter as shown in Fig. 2 (b-d). The results are shown in Table I.

Considering a standard inverter shown in Fig. 2 (a), the simulation results for the $130 \mathrm{~nm}$ technology shown in Table I reveals that applying PTS on only PMOS transistors (column P-PTS) of each inverter in the ring oscillator reduces the frequency of oscillation by $3 \%$. On the other hand, applying PTS on only NMOS transistors (column N-PTS) improves the frequency by $120 \%$, while applying PTS on both NMOS and PMOS transistors increases the frequency only by $100 \%$. This result is expected as the COC ratio corresponding to the PMOS transistor of $8 \mu \mathrm{m}$ width (point E in Fig. 1 (b)) is more than the COC ratio compared to $160 \mathrm{~nm}$ (point C in Fig. 1 (b)), and the NMOS transistor at the width of $4 \mu \mathrm{m}$ (point D in Fig. 1 (a)) has much lower $\mathrm{COC}$ ratio compared to point $\mathrm{A}$. Therefore, in this case, the optimum sizing

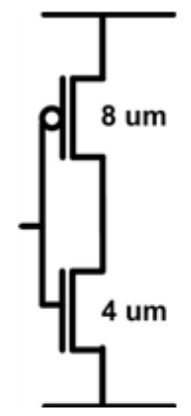

(a) Standard, no PTS

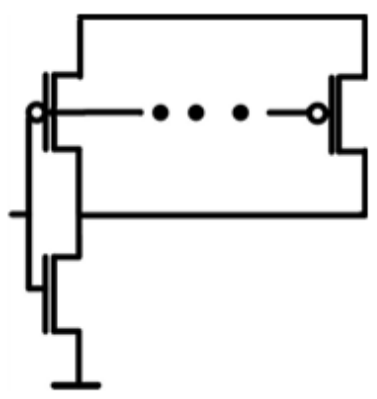

(b) PMOS only PTS (P-PTS)

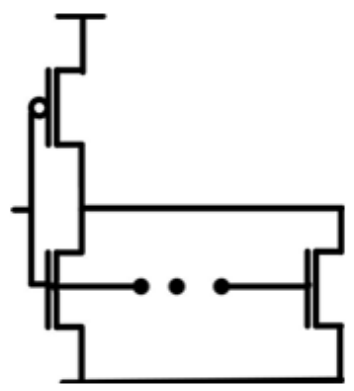

(c) NMOS only PTS (N-PTS)

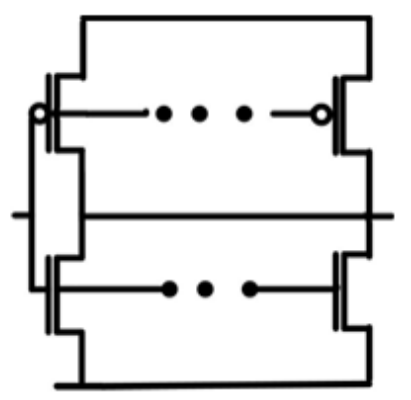

(d) PMOS and NMOS PTS (PN-PTS)

Fig. 2. Inverter configurations for a 19-inverter ring oscillator: (a) Standard, (b) PMOS PTS, (c) NMOS PTS, (d) PMOS and NMOS PTS. 
Table I. Frequency, Delay, and Power of 19-stage ring oscillator and a 4-bit comparator in 130 and $90 \mathrm{~nm}$ technology with the standard, only PMOS PTS (P-PTS), only NMOS PTS (N-PTS), both NMOS and PMOS PTS (PN-PTS), and PTS based on the proposed methodology (OPT-PTS).

\begin{tabular}{|c|c|c|c|c|c|c|}
\hline Technology & Metrics & Standard & P-PTS & N-PTS & PN-PTS & OPT-PTS \\
\hline \multicolumn{7}{|c|}{ Ring Oscillator } \\
\hline \multirow[t]{2}{*}{$130 \mathrm{~nm}$} & Frequency $(\mathrm{KHz})$ & 364.8 & 357.2 & 806.1 & 753.9 & 806.1 \\
\hline & Power (nW) & 10.3 & 9.821 & 23.45 & 21.73 & 23.45 \\
\hline \multirow[t]{2}{*}{$90 \mathrm{~nm}$} & Frequency $(\mathrm{MHz})$ & 6.4 & 5.3 & 11.6 & 9.15 & 11.6 \\
\hline & Power $(\mathrm{nW})$ & 165.9 & 138.4 & 327.6 & 269.5 & 327.6 \\
\hline \multicolumn{7}{|c|}{ 4-bit Comparator } \\
\hline \multirow[t]{2}{*}{$130 \mathrm{~nm}$} & Delay(ns) & 908.3 & 862.0 & 573.5 & 548.3 & 531.0 \\
\hline & Power (pW) & 772 & 768.6 & 1401 & 1403 & 1390 \\
\hline \multirow[t]{2}{*}{$90 \mathrm{~nm}$} & Delay(ns) & 56.0 & 74.7 & 36.2 & 48.6 & 34.2 \\
\hline & Power (nW) & 9.76 & 8.32 & 17.12 & 15.2 & 16.79 \\
\hline
\end{tabular}

(column OPT-PTS) is achieved by applying PTS on NMOS transistors, and avoiding applying PTS on PMOS transistors. Note that by applying PTS the power consumed by the ring oscillator increases by $127 \%$.

In the $90 \mathrm{~nm}$ technology, the COC ratio for both transistor types follow the same behavior as that of the $130 \mathrm{~nm}$ technology. Simulation illustrates that the corresponding COC ratio of the NMOS transistor versus width has a sharp decay, whereas the COC ratio corresponding to the PMOS transistor increases after a certain width $(1 \mu \mathrm{m})$. The simulation results on a 19-stage ring oscillator shown in Table I for the $90 \mathrm{~nm}$ confirm that applying PTS on all PMOS transistors of $8 \mu \mathrm{m}$ width results in $16 \%$ lower frequency compared to that of the standard sizing. On the other hand, applying PTS on only NMOS transistors improves the speed almost by $81 \%$. Applying PTS on both PMOS and NMOS transistors increases the speed only by $41 \%$ compared to the standard case. Again here, for achieving the maximum speed we advise applying PTS just on NMOS transistors and not PMOS transistors. As shown in Table I, the power consumed by the ring oscillator increases compared to the standard case from $165.9 \mathrm{nW}$ to $327.6 \mathrm{nW}$.

We also applied our methodology on implementing a 4-bit comparator. The simulation results after Logical Effort optimization are shown in Table I (column Standard). 
By running Logical Effort on this complex circuit, it suggests PMOS transistors with widths in both regions (Fig. 1 (b)). According to our methodology, for NMOS transistors, as all suggested widths have lower COC ratio than the minimum width, we apply PTS on all NMOS transistors in both CMOS technologies. Applying PTS on PMOS transistors depends on whether the Logical Effort locates the width sizes in Region 1 or Region 2. Regardless the fact that PTS should be applied on certain transistors, in Table I we list the simulation results of applying PTS only on all PMOS and only on all NMOS transistors in the P-PTS, and N-PTS columns, respectively. Column $P N$ $P T S$ lists the simulation results for applying PTS on both NMOS and PMOS transistors. The last column, OPT-PTS, illustrates the results of optimum sizing (maximum speed) based on our methodology. This column shows $41 \%$ increase of speed compared to the standard sizing, which is $3 \%$ more than applying PTS on all transistors blindly. The reason that our methodology in this case doesn't show considerable improvement compared to the blind PTS is that most of the PMOS transistors are in Region 1.

The result of applying the proposed methodology, i.e. selective application of PTS, on the 4-bit comparator in the $90 \mathrm{~nm}$ technology is also shown in OPT-PTS column of Table I. The speed is increased by around $39 \%$ compared to standard sizing, while applying blind PTS only improves the speed by $13 \%$. Note that applying PTS only on all PMOS transistors (P-PTS) degrades the speed by $33 \%$, while applying PTS only on all NMOS transistors (N-PTS) improves the speed by $35 \%$ compared to the standard sizing.

The power consumed by the 4-bit comparator in both $130 \mathrm{~nm}$ and $90 \mathrm{~nm}$ technologies shows $80 \%$ and $72 \%$ increase when PTS is applied compared to the standard case.

\section{Conclusion}

Although using parallel transistor stacks (PTS) gives rise to considerable speed benefit in the subthreshold circuits, there are cases where PTS should be avoided. In this paper we proposed a methodology to advise selective application of PTS on some transistors in a circuit. Applying the proposed methodology on different circuits illustrates up to $40 \%$ increase in speed compared to when PTS is applied on all transistors blindly. Incorporating our methodology shows $38 \%$ to $121 \%$ speed improvement compared to the conventional transistor sizing optimization method. Besides, it is important to note that using PTS increases the circuit area up to $50 \%$ and according to our simulations the power consumption is linearly proportional to the speed. 